\title{
Development of an Automatic Bidirectional Wireless Charging System for Mobile Devices
}

\author{
James Washak \\ College of Engineering, \\ Mathematics and Physical \\ Sciences \\ University of Exeter \\ Exeter, United Kingdom \\ jw785@exeter.ac.uk
}

\author{
Cristina Alexandru \\ College of Engineering, \\ Mathematics and Physical \\ Sciences \\ University of Exeter \\ Exeter, United Kingdom \\ ca410@exeter.ac.uk
}

\author{
Dibin Zhu \\ College of Engineering, \\ Mathematics and Physical \\ Sciences \\ University of Exeter \\ Exeter, United Kingdom \\ d.zhu@exeter.ac.uk
}

\begin{abstract}
Bidirectional wireless charging technology is an emerging technology within the wireless power transfer (WPT) field of research. Bidirectional wireless power transfer (BWPT) allows the same device to not only receive power but also transmit power to other compatible devices. This paper documents the development of a prototype BWPT system for mobile devices which will allow for two mobile devices to share power wirelessly without the need for cables. In this device the data transfer capabilities of WPT will be utilized to ensure that minimal input is required from a potential user by automating the power transfer process as much as possible to make the system simple to use for any potential end-user. This feature will differentiate this design from other existing BWPT where this feature seems to be ignored or manual inputs from the user are required to configure the devices involved in BWPT.
\end{abstract}

Keywords-bidirectional; wireless charging; wireless power transfer (WPT); bidirectional wireless power transfer (BWPT); amplitude shift keying (ASK)

\section{INTRODUCTION}

Wireless power transfer (WPT) is technology that allows electrical power to be transferred to electronic devices without the need for any physical connection [1]. Bidirectional wireless power transfer (BWPT) is a form of WPT, but in BWPT the hardware is designed so that power can be transmitted in either direction so that a device could not only receive power but also send it if necessary. Today WPT technology is now utilized in commercial applications primarily to charge small electronic devices, while BWPT is a much newer technology and therefore has made limited inroads into the commercial sector as of the end of 2018.

Currently, a review of existing information about BWPT including academic papers, commercial products and patents reveals that although the process of BWPT has been demonstrated in a technical and theoretical sense, issues revolving the user experience and its practicality have not been comprehensively addressed as of now. None of the existing research has even addressed the issues of data transfer and so in turn completely ignoring any control signals that would needed, so that the process could be automated as much as possible. Automated BWPT is required to make the technology practical and easy to use for potential end-user. A patent by Cook et al.
[2] did mention data transfer in the context of BWPT. The patent proposed the design of a BWPT system [2]. In this patient the issue of data transfer was raised but many are context relating transferring user data such as photos rather than control signals used to monitor the charging process. The only commercial application of BWPT thus far is the Huawei Mate 20 Pro which came to market in late 2018, this product does allow the phone to transmit power to other devices [3]. However, the user must select direction of the power transmission via the settings menu.

The aim of this project is to develop a prototype device to demonstrate that BWPT between electronic devices which communicate with each other during the process can be used to highly automate the BWPT process. This feature can hence make the entire process of BWPT appear seamless to any potential end-user. The proposed system would require the enduser to simply place the two devices' induction coils on top of each other and the system would then automatically determine which device will be transmitting power and which device will be receiving power, based on a set of parameters which could be changed depending on the user's preference. This would remove the need for the user to have to configure any settings manually therefore increasing the ease of use and practicality of this technology, compared to existing solutions.

\section{SYSTEM DESIGN}

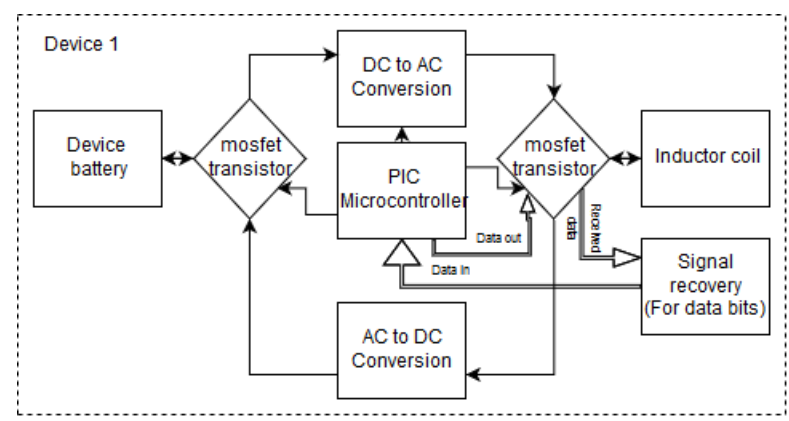

Fig. 1. - Block diagram of designed system

Fig. 1 shows a block diagram representation of the designed system. At the heart of the system is a microcontroller which manages all the various sub elements of the circuit. One of its primary tasks is to trigger the correct transistor switches so the 
required circuit element is selected when needed. Its second key role is to process the data that has been received and to transmit data to be second device.

Some crucial processes such as DC-AC conversion require a high frequency square wave input from the microcontroller, while the microcontroller also triggers various base legs of transistors which act as switches, which allow various subsections of the circuit to be selected depending on what is needed: sending power, receiving power, receiving data or sending data.

The microcontroller is programmed to manage the hardware during the charging process. The flowchart in Fig. 2 shows an overview of how the program works. The communication is half-duplex, i.e. the communication medium can only support communication in a single direction at one time, so the two devices will need to take turns when communicating. Before each device is connected, each device sends out a request for response at random intervals. When it is not sending these messages, it is listening for a response from the other device. If one device receives a request to respond it will send an acknowledgement back and in this case from this point only the device which sent the request for an acknowledgement will initiate communication in future. The device which sent the acknowledgement in future will only respond to requests from the other device for information, this way the risks of data collision within the communication medium will be limited.

Once communication has been established information such as battery level data is shared and the device in charge of requesting data will decide which device should receive power and which device should send power. This information is transmitted to the other device and from here the power transfer in the appropriate direction occurs. At regular intervals the device which request data will check the two devices are still connected and continue the power transfer if this is the case, otherwise the power transfer stopped and the entire cycle repeats.

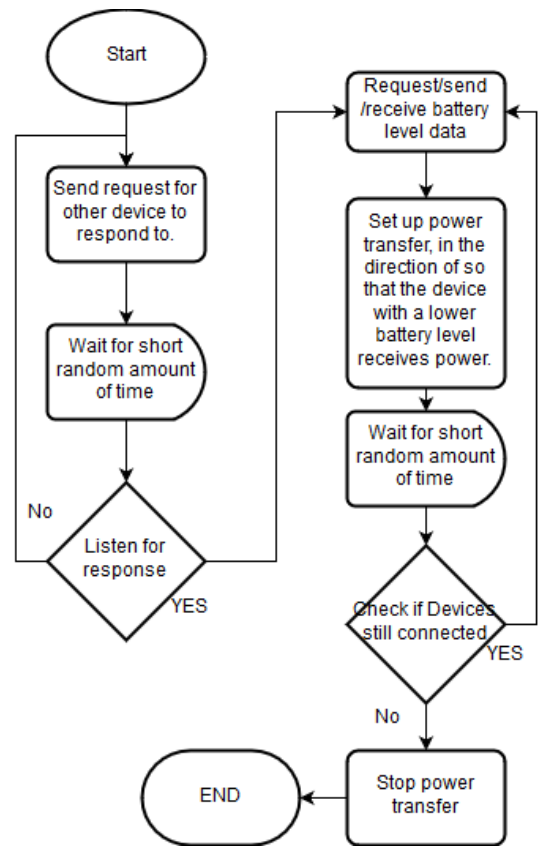

Fig. 2. Flow chart for program that will manage the system.

\section{PRELIMINARY TEST}

Fig. 3 shows the current prototype of the system that was developed and is annotated to show the key elements from the block diagram. Two identical prototypes were built so that they could be comprehensively tested.

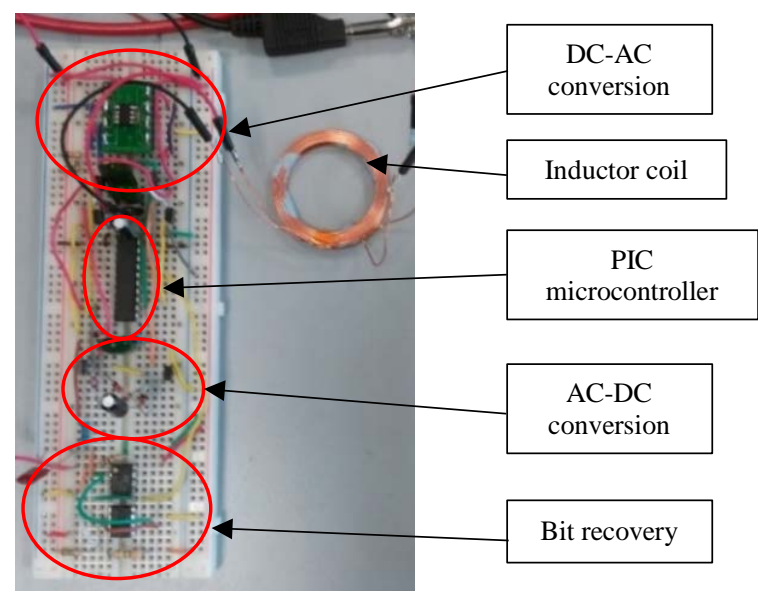

Fig. 3. prototype circuit developed

The system has been simulated in Multisim and implemented on a breadboard. The current design achieved all the required functionality and preliminary tests have shown that the hardware is fully functional with the ability to transmit binary data and power in both directions between two prototype devices.

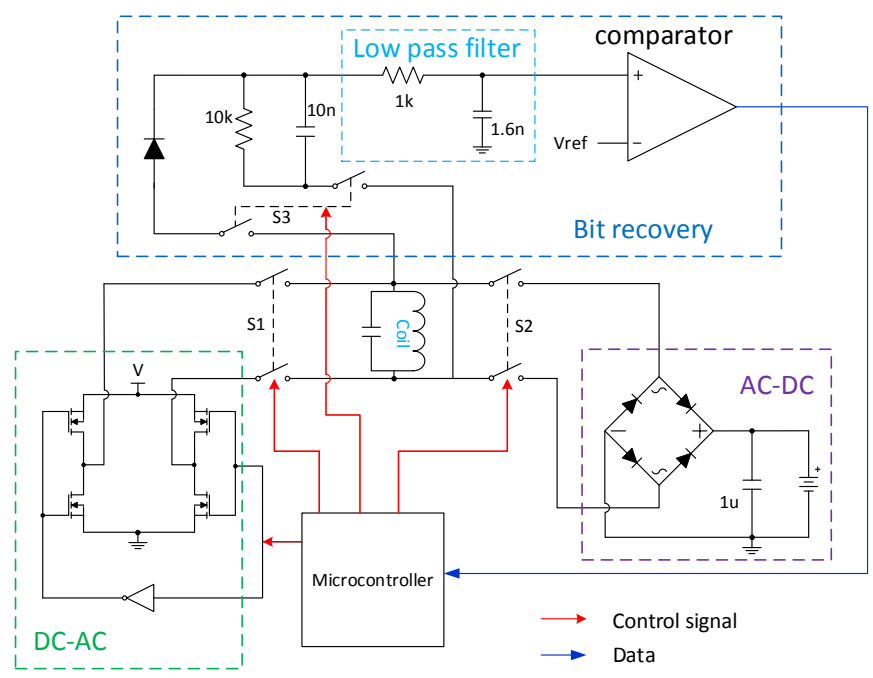

(a) Full Circuit diagram of designed system.

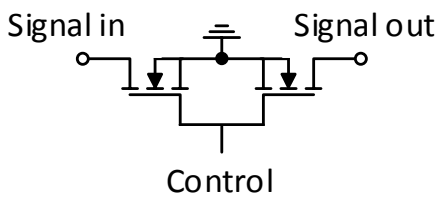

(b) MOSFET based AC switch.

Fig. 4. Circuit diagram. 
Fig. 4a show the complete circuit including the microcontroller which is responsible for the sending of various control signals and receive data. The system consists of three components, i.e. DC-AC module which is used on the transmission side as well as AC-DC module and bit recovery module which are used on the receiving side. These three modules share the same coil. Three sets of MOSFET based AC switches are used to select the relevant module. Closing S1, S2 and S3 are to select DC-AC module, AC-DC module and bit recovery module respectively. Fig. 4b shows the detail of the MOSFET based AC switch. It consists of two N-channel MOSFET. The two source terminals are connected to the ground. The two gate terminals are connected together as the control terminal. When a logic high signal is applied, the switch is on (closed). When a logic low signal is applied, the switch is off (open). The two drain terminals are terminals of the AC switch. Details of this switch and its benefit over relay switches can be found in [4].

The system uses amplitude phase key shifting (ASK) modulation to encode digital data into an analogue form so it can be transmitted across the existing analogue communication medium. The carrier signal in this case is the $125 \mathrm{kHz}$ AC current generated by the transmitting device, which is also used to transfer power. The bit recovery circuit is designed to decode the ASK modulated signal which contains digital data bits into a digital square wave from the analogue AC signal received. This signal is then inputted into the microcontroller.

\section{TEST RESUlTS}

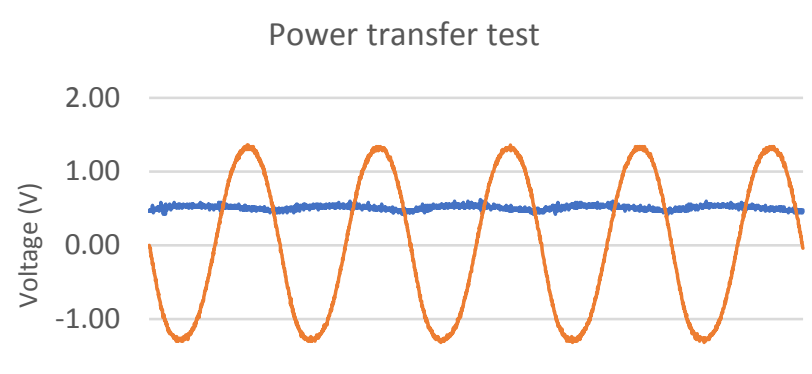

$-2.00$

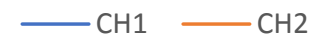

Fig. 5. - Result of power transfer test

Power transfer test with coil misaligned by $20 \mathrm{~mm}$

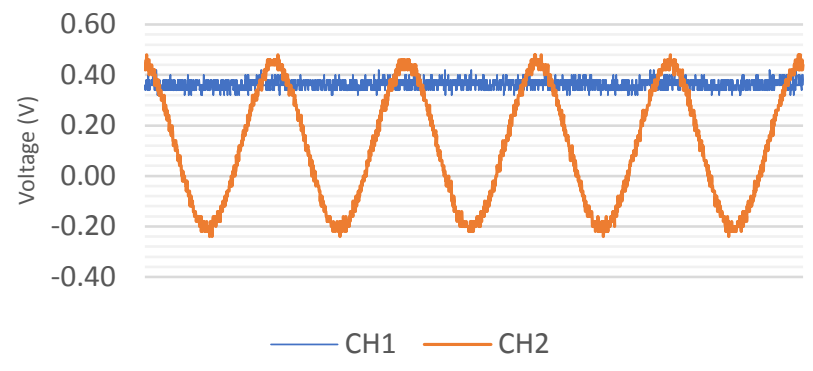

Fig. 6. Result of power transfer test with coil misaligned by $20 \mathrm{~mm}$
Figs. 5 and 6 show evidence of successful power transfer between the two prototype devices and that the can successfully convert the received AC signal into a DC output. $\mathrm{CH} 1$ measured the DC output on receiving board its conversion from AC to DC, while $\mathrm{CH} 2$ measured the $\mathrm{AC}$ signal directly from the receiving coil, this signal was then inputted into the AC to DC conversion circuit. For this test the MOSFET transistors were configured to send the received signal to the AC-DC conversion circuit.

The tests were repeated with varying amounts of misalignment between the coils to ensure that power transfer could still occur in scenarios where the coil was not perfectly aligned. The test showed that up to $20 \mathrm{~mm}$ horizontal coil misalignment as shown in Fig. 7 could be achieved with minimal power loss although beyond this there was a sudden decrease in power loss.

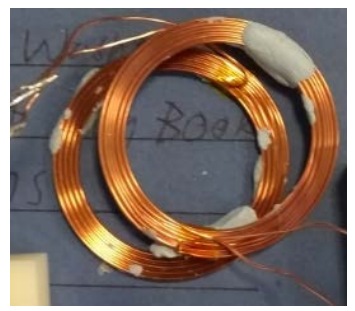

Fig. 7. Image of coil misaligned by $20 \mathrm{~mm}$ horizontally.

Data transfer test " $\mathrm{C}$ " ASCII character (01000011)

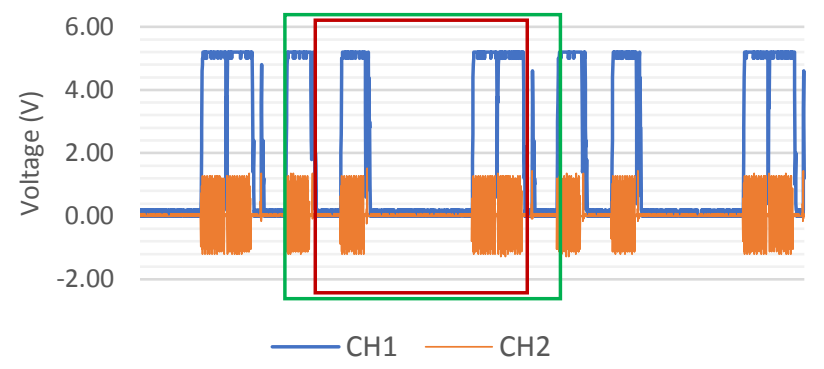

Fig. 8. Data transfer test “C” ASCII character (01000011)

Data transfer test "C" ASCII character close-up on inputs into op-amp comparator

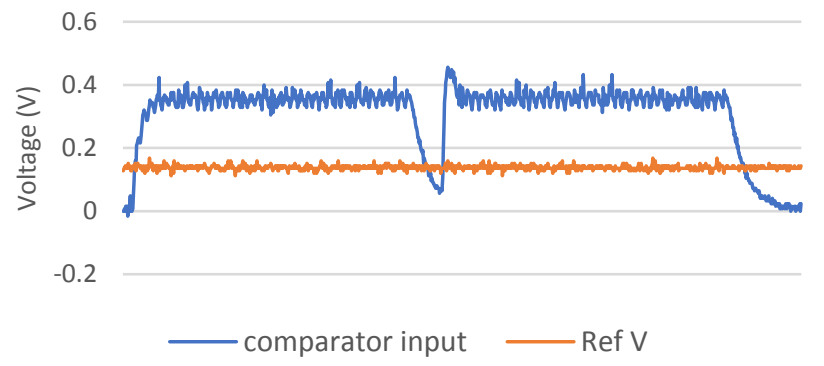

Fig. 9. Data transfer test “C” ASCII character (01000011) close-up on inputs into op-amp comparator for two " 1 " bits.

Fig. 8 shows the results of the data transfer test which involves repeatedly 8-bit binary values to ensure the correct bits were being sent and received. The bits highlighted in red represent the data bits while the bits surrounded by the green line 
are the stop and start bits. CH1 shows the signal that is inputted into the receiving microcontroller and $\mathrm{CH} 2$ shows the signal received directly from the coil before bit recovery. Fig. 9 show the final stage of the bit recovery circuit where the received analogue signal is inputted into a comparator which is designed to have a reference voltage close to $0.1 \mathrm{~V}$. Although practice this value was closer to $0.14 \mathrm{~V}$. This causes the high bits to be amplified to create the $5 \mathrm{~V}$ square wave digital output seen in Fig. 8. Before the signal is inputted into the comparator it passes through a diode and a low pass filter to remove the negative current and signals with a frequency less than $100 \mathrm{kHz}$, as the received signal should have a frequency of $125 \mathrm{kHz}$. The peak voltage of the final signal that is inputted into the comparator show signs it has attenuated to approximately half its original value, $0.4 \mathrm{~V}$ from $0.9 \mathrm{~V}$.

The system uses amplitude phase key shifting (ASK) modulation to allow for the digital data to be sent across the existing analogue medium. The received signal is sent to a circuit that decodes the analogue signal and outputs a 5V square wave. Analysis of the bits receive shows that the correct data bits were sent. In later versions the program loaded on the PIC microcontroller added a " 0 " parity bit for odd parity in addition to the high start bit and low stop bit to each 8-bit packet of data. The tests in Figs. 8 and 11 showed additional start and stop bits were present. The parity bit alongside the correct data bits and stop/start bit can be seen in Fig. 10. For these tests the MOSFET transistors were configured to send the received signal to the bit recovery circuit.

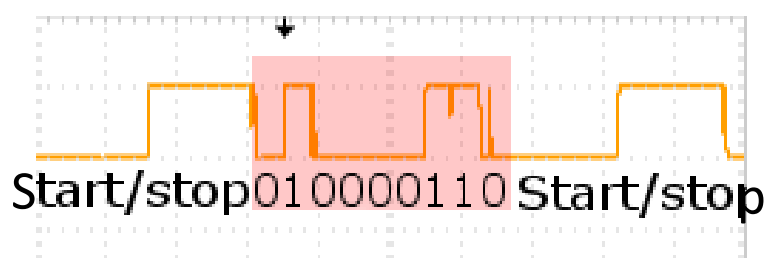

Fig. 10. Data transfer test “C” ASCII character (01000011) with 0 parity bit.

Each bit had a time period of 220us which gives the system a data rate of 4545 bits per second (approximately 4.5 kbits/second). It should be noted 3 out of 11 bits per packet contain no useful data and therefore only approximately $73 \%$ of the bits sent contain real data, therefore the actual data rate would be closer to $3.2 \mathrm{kbits} / \mathrm{second}$. This rate could easily be adjusted by changing the time period allocated to each bit.

\section{Data transfer test "C" ASCII character (01000011) with $15 \mathrm{~mm}$ of coil misalignment}

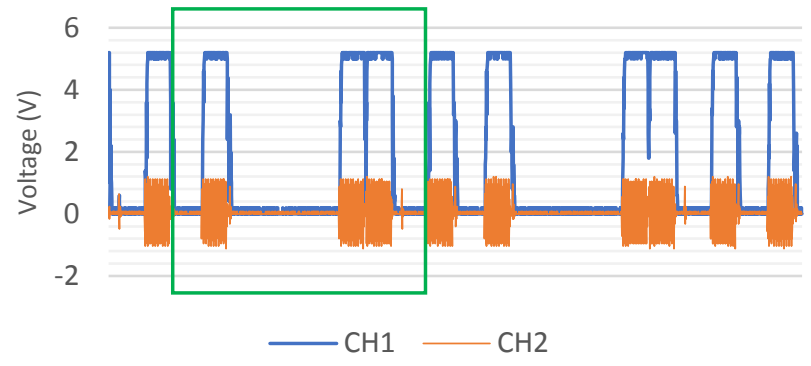

Fig. 11. data transfer test "C" ASCII character (01000011) with $15 \mathrm{~mm}$ of coil misalignment. Green box highlights one set of start/stop and data bits.
The tests were repeated with varying amounts of misalignment between the coils. Fig. 11 shows the result of a test done with $15 \mathrm{~mm}$ of coil horizontal misalignment, which found that accurate data transfer would still occur at this point. Further tests showed that any misalignment beyond this level would result in inaccurate data transfer, an example of this can be seen in Fig. 12.

Fig. 12 shows a test done with $17 \mathrm{~mm}$ of coil misalignment and at this point the received signal is too attenuated and therefore the decoder circuit is unable to restore the signal to its original digital form. In addition, as mentioned before some attenuation of the signal also occurs when it is passed through the early stages of the bit recovery circuit. Consequently, the final signal inputted into the comparator is never greater than the reference voltage $0.14 \mathrm{~V}$ therefore no high bits can be outputted. In theory the system tolerance to coil misalignment error could be improved by increasing the reference voltage or improving the efficiency of the power transfer process to thus increase the voltage of the received signal, to ensure there is a greater chance of the received signal being greater than the reference voltage.

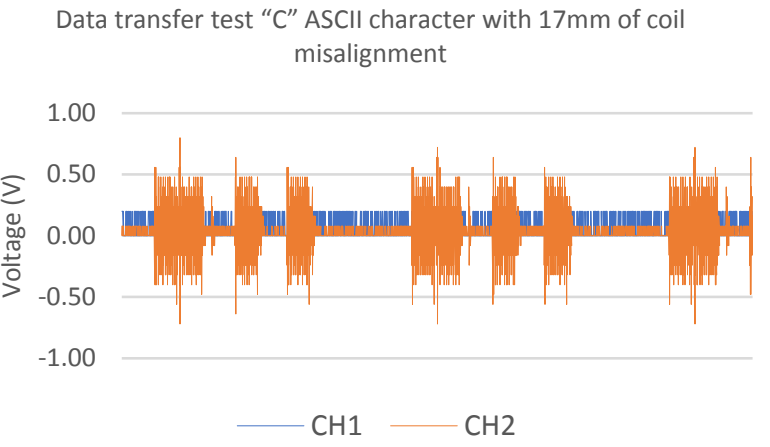

Fig. 12. data transfer test "C" ASCII character with $17 \mathrm{~mm}$ of coil misalignment.

The program was designed to be interrupted when it detected the high start bit at input pin (RC0 on the PIC18F1XK50), this pauses any existing task the program is coming out so that the input voltage level can be sampled to save the bits from the square wave in the program's memory. The parity bit can be checked to locate single bit errors. After the Interrupter the program will return to same point it was at before the interrupter occurred. The hardware is limited in the fact that both data and power cannot be transferred at the same time however the as over 3000 bits or approximately 400 ASCII character per second can be transmitted. Because of this the time required to send data is minimal therefore the majority of the system's time can be devoted to power transfer. Consequently, BWPT system with data transfer functionality would appear to the average consumer to be transferring power constantly even if periodic bursts of data were exchanged between the devices.

The test data shows that a BWPT system can facilitate both power transfer and reliable data transfer in both directions between the two involved devices. 


\section{ESTABLISHING COMMUNICATION AND COMMUNICATION PROTOCOLS}

The hardware developed created a half-duplex communication medium for data to be transferred between both devices. Half duplex communication mediums can only facilitate the transfer of information in one direction at a time and subsequently successful communication is dependent on each device waiting for its turn. A master slave relationship is used to overcome this barrier. Before a connection is established both devices randomly send out requests to ask for a connection to be established, when this is not being done it listens to see if a request has been sent. If a device receives a request for a connection to be established, they become the slave device while the sender is the master. The slave responds to notify the master device that it is connected. From this point only the master device will send out information while the slave simply responds to requests for data from the master. This ensures there is minimal chance of data collisions as the slave device only transmits data in response to requests from the master. Once a connection is established the devices exchanged battery level data and the master then determines the direction of power transfer, after which the hardware is configured on both devices using the MOSFET transistor switches and the transfer of power begins. This process stops when the devices are no longer in range or the charging devices power battery level falls below a user-defined threshold.

The user may remove either of the devices at any time during the charging process which means the devices must regularly check that they are still in range. To achieve this the master device sends a request for an acknowledgement at regular intervals, which the slave must respond to, so that the master device knows it's still in range of the slave device. In addition, the slave device also will assume that the master device is out of range if it does not receive an acknowledgement with in a given period, the countdown is reset each time an acknowledgement is received and sent. This system ensures that the devices know as soon as possible when the connection between them is lost at which point, they can stop the power transfer process and return to the start of the program to begin the cycle again.

\section{CONCLUSIONS AND FUTURE WORKS}

This paper presents a fully automated bidirectional wireless power transfer system, with data transfer capabilities. When two devices are coupled, they are able to exchange information about battery level. Based on this information, the device with higher battery level will then transmit energy wirelessly to the other device until both devices reach similar battery levels. It is worth mentioning that the proposed system is suitable for any types of portable devices.

The tests have demonstrated that the prototype can achieve all targeted functions which are listed below:

- $\quad$ Reliably transmit 8bit binary data between devices.

- The rate of data transfer is sufficient for the target use.

- $\quad$ Transmit DC power between both devices.

- $\quad$ Able to detect signals from other devices.

- MOSFET transistor switches allow program to automatically configure hardware e.g. setup for receiving power or sending power.

- $\quad$ Power and data transfer can tolerate small amounts of horizontal coil misalignment less than $15 \mathrm{~mm}$.

Future work would be to improve and the design of the BWPT hardware in order to improve the efficiency of power transfer.

\section{REFERENCES}

[1] S. Miura, K. Nishijima and T. Nabeshima, "Bi-directional Wireless Charging between Portable Devices," in International Conference on Renewable Energy Research and Applications, Madrid, 2013.

[2] N. P. Cook, L. Sieber and H. Widmer, "Bidirectional wireless power transmission”. United states of America Patent US20100148723A1, 2010.

[3] Huawei Mate 20 Pro [Online]. Available: https://consumer.huawei.com/en/phones/mate20-pro/. [Accessed 26 April 2019].

[4] C. A, Alexandru and D. Zhu, " Comparisons of MOSFET and Relay Switches in Impedance Matching Networks for Wireless Power Transfer," in IEEE Wireless Power Transfer Conference 2019, London, 2019 